\title{
Lattice distortion and electronic structure of magnesium-doped nickel oxide epitaxial thin films
}

\author{
Yanna Chen, ${ }^{1,2}$ Osami Sakata,,${ }^{1,2,3,{ }^{*}}$ Ryosuke Yamauchi, ${ }^{3}$ Anli Yang, ${ }^{1}$ Loku Singgappulige Rosantha Kumara, ${ }^{1}$ \\ Chulho Song, ${ }^{1}$ Natalia Palina, ${ }^{1}$ Munetaka Taguchi,${ }^{4}$ Toshiaki Ina, ${ }^{5}$ Yoshio Katsuya,,${ }^{1}$ Hiroshi Daimon, ${ }^{4}$ \\ Akifumi Matsuda, ${ }^{3}$ and Mamoru Yoshimoto ${ }^{3}$ \\ ${ }^{1}$ Synchrotron X-ray Station at SPring-8, Research Network and Facility Services Division, National Institute for Materials Science (NIMS), \\ 1-1-1 Kouto, Sayo-cho, Sayo-gun, Hyogo 679-5148, Japan \\ ${ }^{2}$ Synchrotron X-ray Group, Research Center for Advanced Measurement and Characterization, NIMS, 1-1-1 Kouto, Sayo-cho, Sayo-gun, \\ Hyogo 679-5148, Japan \\ ${ }^{3}$ Department of Materials Science and Engineering, School of Materials and Chemical Technology, Tokyo Institute of Technology, \\ 4259 Nagatsuta-cho, Midori-ku, Yokohama 226-8502, Japan \\ ${ }^{4}$ Department of Material Science, Nara Institute of Science and Technology (NAIST), 8916-5 Takayama-cho, Ikoma, Nara 630-0192, Japan \\ ${ }^{5}$ Research \& Utilization Division, Japan Synchrotron Radiation Research Institute (JASRI), 1-1-1 Kouto, Sayo-cho, Sayo-gun, \\ Hyogo 679-5198, Japan
}

(Received 31 August 2016; revised manuscript received 13 March 2017; published 1 June 2017)

\begin{abstract}
Magnesium-doped nickel oxide $\left(\mathrm{Mg}_{x} \mathrm{Ni}_{1-x} \mathrm{O}\right)$ thin films are transparent over a wide ultraviolet-visible spectral range and over a wide $\mathrm{Mg}$ content range. However, the influence of the $\mathrm{Mg}$ dopant on the structure and properties of $\mathrm{NiO}$ films is poorly understood. In this work, the lattice distortion and the electronic structure of $\mathrm{Mg}_{x} \mathrm{Ni}_{1-x} \mathrm{O}$ $(0 \leqslant x \leqslant 0.52)$ thin films deposited on ultrasmooth sapphire substrates were investigated using synchrotron $\mathrm{x}$ rays. Films with higher $\mathrm{Mg}$ content had lower values of Debye temperature and atomic order parameter. The nearest $\mathrm{Ni}-\mathrm{O}$ distance and the in-plane nearest $\mathrm{Ni}-\mathrm{Ni}$ distance both expanded with increasing $\mathrm{Mg}$ content. The Ni $2 p$ core-level spectra and the valence band spectra of the $\mathrm{Mg}_{x} \mathrm{Ni}_{1-x} \mathrm{O}$ thin films showed complex multiplet structures that were caused by the strong electron correlation in the Ni $3 d$ states, where the spectral features are strongly dependent on both the distortion of the $\mathrm{NiO}_{6}$ octahedra and the $\mathrm{Mg}$ content. We found that the electronic structures are mainly a result of hybridization of $\mathrm{Ni} 3 d$ and $\mathrm{O} 2 p$ in the $\mathrm{NiO}_{6}$ octahedra and the reduction of the Zhang-Rice bound state following $\mathrm{Mg}$ doping. Finally, the flexibility of the band gap tuning in $\mathrm{Mg}_{x} \mathrm{Ni}_{1-x} \mathrm{O}$ thin films is explained.
\end{abstract}

DOI: 10.1103/PhysRevB.95.245301

\section{INTRODUCTION}

Nickel oxide $(\mathrm{NiO})$ with a rock-salt structure is a wideband-gap $p$-type semiconductor that shows excellent stability in atmospheric air, and has high crystallinity and transparency. The multifunctional properties of $\mathrm{NiO}$ make it useful for a variety of applications, including ultraviolet (UV) detectors [1], high durability hole transport layers in organic solar cells [2], electrochromic devices [3], and UV light-emitting diodes [4]. To improve the performance of the material, $\mathrm{NiO}$ films have been doped using a wide range of elements, with a particular focus on the roles of monovalent ions or trivalent ions as acceptor or donor species to enhance film conductivity $[5,6]$. In addition, transition metals with $3 d$ electrons are also attractive as dopant materials for $\mathrm{NiO}$ because of their rich ranges of dielectric and magnetic properties [7-9]. When compared with these elements, elemental magnesium has the same valence and similar ionic radii to those of $\mathrm{Ni}$ [10]. However, magnesium-doped nickel oxide $\left(\mathrm{Mg}_{x} \mathrm{Ni}_{1-x} \mathrm{O}\right)$ offers great flexibility in terms of both dopant content and band gap tuning.

Usually a phase transformation is observed in metal oxides with high dopant content. For example, lithium-doped nickel

\footnotetext{
*SAKATA.Osami@nims.go.jp

Published by the American Physical Society under the terms of the Creative Commons Attribution 4.0 International license. Further distribution of this work must maintain attribution to the author(s) and the published article's title, journal citation, and DOI.
}

oxide $\mathrm{Li}_{x} \mathrm{Ni}_{1-x} \mathrm{O}$ undergoes a phase transformation from cubic to a rhombohedral structure at $x=0.31$; as a result, only lightly doped materials can be used in the devices described above $[11,12]$. However, previous studies indicated that the lattice tended to expand in polycrystalline $\mathrm{Mg}_{x} \mathrm{Ni}_{1-x} \mathrm{O}$ thin films over a wide $\mathrm{Mg}$ dopant content range from $x=0$ to $x=1[13,14]$. The effects of the $\mathrm{Mg}$ dopant in these polycrystalline thin films on the lattice parameters were on the preparation process used. As a result, it is difficult to evaluate the role played by $\mathrm{Mg}$ as a dopant in $\mathrm{NiO}$ [14-16]. For this reason, we have investigated high-quality $\mathrm{Mg}_{x} \mathrm{Ni}_{1-x} \mathrm{O}$ epitaxial thin films in this work to determine the intrinsic effects of $\mathrm{Mg}$ on the $\mathrm{NiO}$ lattice. The Debye temperature $\left(\Theta_{M}\right)$ is a fundamental characteristic structural parameter of a solid and describes the dynamic motions of the atoms within the material. $\Theta_{M}$ represents not only a measure of the hardness of a crystal in the Debye model, which is used to estimate the phonon contribution to specific heat, but also provides a quantitative evaluation of both the lattice distortion and the atomic-scale disorder. A number of physical parameters of materials, including the mean-square atomic displacement and the elastic constant, are related to $\Theta_{M}[17,18]$.

With their high capacity for binary metals in the oxide structure and excellent stability, $\mathrm{Mg}_{x} \mathrm{Ni}_{1-x} \mathrm{O}$ thin films offer tunable optical transmittance over a wide $\mathrm{Mg}$ content range [13-16]. Using a low-cost sol-gel spin coating method, $\mathrm{Mg}_{x} \mathrm{Ni}_{1-x} \mathrm{O}$ thin films with a full $x$ range were prepared. The optical transmittance of these films in the UV-visible spectral region reached as high as $90 \%$ and the estimated energy gap 
of these thin films could be tuned from 3.5 to $4.4 \mathrm{eV}$ for values of $x=0.0$ to 0.77 [14]. Density functional theory calculations of the $\mathrm{Ni}_{x} \mathrm{Mg}_{1-x} \mathrm{O}$ band structure showed that the band gap evaluation is mainly dependent on changes in the deep $\mathrm{Ni} 3 d$ orbitals in the $\mathrm{MgO}$ band gap [19].

As the combination of a configuration interaction model with photoemission spectroscopy results verified, the energy band gap and the main spectral peaks in the classical chargetransfer insulator $\mathrm{NiO}$ were determined by the interactions between the neighboring $\mathrm{Ni} 3 d$ states and the hole states of $\mathrm{O} 2 p$, which provide unique nonlocal screening properties and support intersite charge-transfer excitation in $\mathrm{NiO}[20,21]$. The Zhang-Rice doublet bound states were introduced into the configuration interaction model to describe the nonlocal screening behavior and explained the charge transfer effects [22]. The calculations described above were all performed on the basis of the octahedral structure formed by a central nickel atom and the six nearest-neighbor coordinated oxygen atoms $\left(\mathrm{NiO}_{6}\right)$. There are two important points overall related to these $\mathrm{NiO}_{6}$ octahedra. First, there is strong hybridization between nickel $3 d$ and oxygen $2 p$ in the $\mathrm{NiO}_{6}$ octahedron, which is sensitive to the local symmetry of the $\mathrm{NiO}_{6}$ octahedral structure. Second, intersite hopping of electrons or core holes occurs from the $\mathrm{NiO}_{6}$ octahedra to their neighbors, and this forms the nonlocal intersite interaction or nonlocal screening properties. Therefore, electronic behavior of the material is sensitive to lattice distortion. For $\mathrm{Mg}_{x} \mathrm{Ni}_{1-x} \mathrm{O}$ thin films in particular, and in addition to the lattice distortion, the dopant will also disturb intersite hopping because of a lack of $3 d$ electrons in the dopant. To enable the application of $\mathrm{Mg}_{x} \mathrm{Ni}_{1-x} \mathrm{O}$ thin films in electronic devices, a comprehensive investigation of the lattice distortion and the electronic structure of $\mathrm{Mg}_{x} \mathrm{Ni}_{1-x} \mathrm{O}$ epitaxial thin films will provide a fundamental understanding of both these $\mathrm{Mg}_{x} \mathrm{Ni}_{1-x} \mathrm{O}$ epitaxial thin films and other related oxide thin films.

In this article we investigate $\mathrm{Mg}_{x} \mathrm{Ni}_{1-x} \mathrm{O}$ thin films that were epitaxially grown on ultrasmooth sapphire substrates to understand the effects of $\mathrm{Mg}$ dopants on both the lattice distortion and the electronic structure of these films. We discuss the lattice parameters that were obtained using the synchrotron $\mathrm{x}$-ray diffraction (XRD) method including $\mathrm{X}$-ray reciprocal-lattice space mapping (XRSM) and evaluated the $\Theta_{M}$ values and the order of the atomic arrangements in these films. The substitution effect of the $\mathrm{Mg}$ dopants in the Ni sites was supported by the results of analysis of extended $\mathrm{X}$-ray absorption fine structure (EXAFS) data. Additionally, hard $\mathrm{x}$-ray photoelectron spectroscopy (HAXPES) core-level and valence-band spectral measurements and calculations were performed to determine the influence of the Mg dopant on the electronic structures of these thin films. When these results were combined with near edge $\mathrm{X}$-ray absorption fine structure (NEXAFS) data, we were able to reveal the effects of $\mathrm{Mg}$ dopants on the band gap of $\mathrm{Mg}_{x} \mathrm{Ni}_{1-x} \mathrm{O}$ thin films.

\section{EXPERIMENTAL METHODS}

$\mathrm{Mg}_{x} \mathrm{Ni}_{1-x} \mathrm{O}(0 \leqslant x \leqslant 0.52)$ epitaxial thin films were deposited on atomically stepped sapphire (0001) substrates (step height of $2.2 \AA$ ) by pulsed laser deposition at room temperature $(298 \mathrm{~K})$ under oxygen pressure of $1.3 \times 10^{-3} \mathrm{~Pa}$ using a pulsed $\mathrm{KrF}$ excimer laser. The operating wavelength of this laser was $2480 \AA$, the pulse duration was $20 \mathrm{~ns}$, the laser fluence was $2 \mathrm{~J} / \mathrm{cm}^{2}$, and the frequency was $5 \mathrm{~Hz}$. After deposition, the obtained thin films were annealed by rapid thermal annealing in air at a heating rate of $20 \mathrm{~K} / \mathrm{s}$ up to $1373 \mathrm{~K}$ for $60 \mathrm{~s}$ and were subsequently quenched to room temperature. The preparation process for layer matching epitaxial growth was similar to that for the $\mathrm{NiO}$ thin films that were fabricated in previous work [23]. The $\mathrm{Mg}_{x} \mathrm{Ni}_{1-x} \mathrm{O}$ epitaxial thin films were then scanned by dynamic force microscopy (Hitachi NanoNaviReals/Nanocute). The thin film surface slightly reflected the step-and-terrace morphology of the underlying substrate [root mean square (rms) roughness: $1.1 \AA]$. The $\mathrm{Mg} / \mathrm{Ni}$ atomic ratios of the target thin films were determined by inductively coupled plasma atomic emission spectroscopy (Shimadzu ICPS-8100). The thickness of the resulting $\mathrm{Mg}_{x} \mathrm{Ni}_{1-x} \mathrm{O}$ epitaxial thin films determined using a stylus profiler (Veeco DekTak) was approximately $400 \AA$. A UV/VIS spectrophotometer (Jasco V550) was used to measure the optical transmittance of the films in the region from 1900 to $9000 \AA$.

The XRD and HAXPES measurements were performed at the National Institute for Materials Science (NIMS) beamline BL15XU at SPring-8. The measurements were similar to those that were used in Refs. [24,25]. The wavelength $\lambda$ of the incident $\mathrm{x}$ rays in the XRD measurements was $1.000 \AA$. XRSM results showed that the full width at half maximum values around the sapphire $11 \overline{2} 3$ Bragg position along the $q_{T}, q_{R}$, and $q_{\perp}$ directions were $7.5 \times 10^{-1}, 1.5 \times 10^{-1}$, and $1.1 \mathrm{mrad}$, respectively. The angular widths of a $\theta-2 \theta$ scan and a $\theta$ rocking curve were 73 and $34 \mu \mathrm{rad}$, respectively, around the 0006 Bragg position. These values indicate that the experimental measurement resolution was sufficiently reliable. For the HAXPES measurements, a high-resolution hemispherical electron analyzer (VG Scienta R4000) was used. The photon energy was set at $5.95 \mathrm{keV}$ and the total energy resolution was $240 \mathrm{meV}$. The angle between the x-ray direction and the direction at which the photoelectrons enter the analyzer was set at $90^{\circ}$. The take-off angle of these photoelectrons with respect to the sample surface was set at $88^{\circ}$. The probing depth used for these thin films was 216 to $273 \AA$ according to the TPP-2M (Tanuma, Powel, and Penn) formula for an inelastic mean-free path [26]. To determine the electrostatic charging effects due to surface contamination, we monitored the binding energy of the $\mathrm{C} 1 s$ photoemission signal.

The EXAFS measurements were carried out at beamline BL01B1 at SPring-8. A Si (111) double-crystal monochromator was used to perform the Ni $K$-edge measurements. The $\mathrm{X}$-ray fluorescence yield was collected using a 19-element Ge solid-state detector oriented at $90^{\circ}$ with respect to the incident beam. The spectra were recorded from -200 to 800 $\mathrm{eV}$ relative to the edge energies, which were calibrated using $\mathrm{NiO}$ bulk materials for reference. The total energy resolution was $250 \mathrm{meV}$. The EXAFS oscillations $\chi(k)$ were extracted and were analyzed using the Demeter software package [27]. The NEXAFS data were recorded in BL12, the SAGA Light Source. The surfaces of all thin films were cleaned in advance by $\mathrm{Ar}^{+}$bombardment at $500 \mathrm{eV}$ with a current of $0.002 \mu \mathrm{A}$ for $4 \mathrm{~min}$. The total electron yields were collected using a 16-channel multichannel detector. The probing depth for 


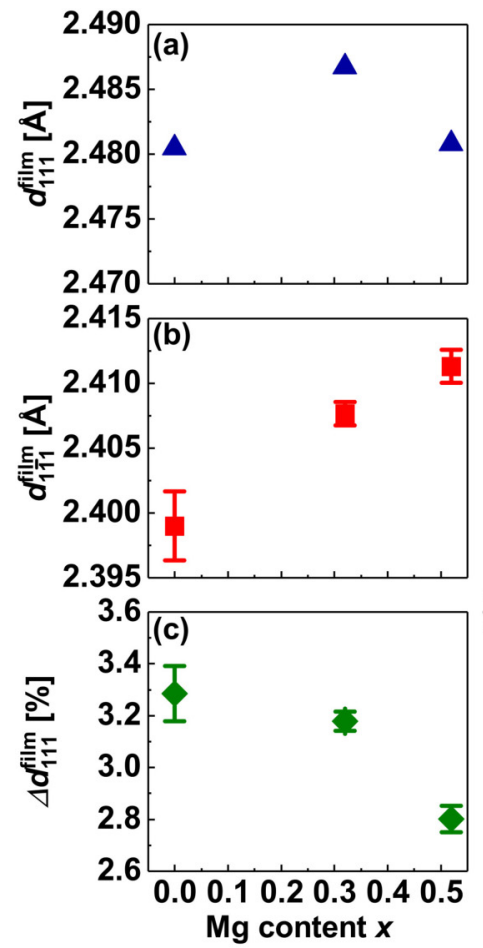

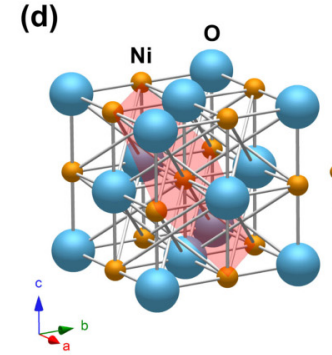

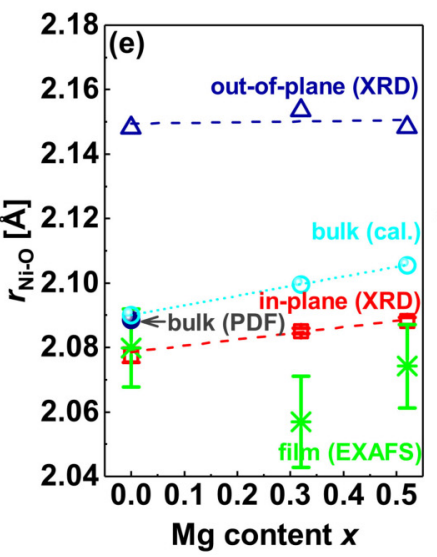

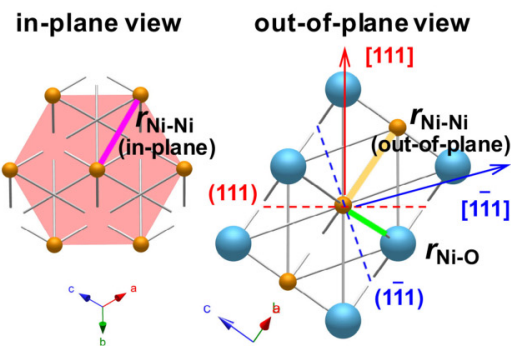

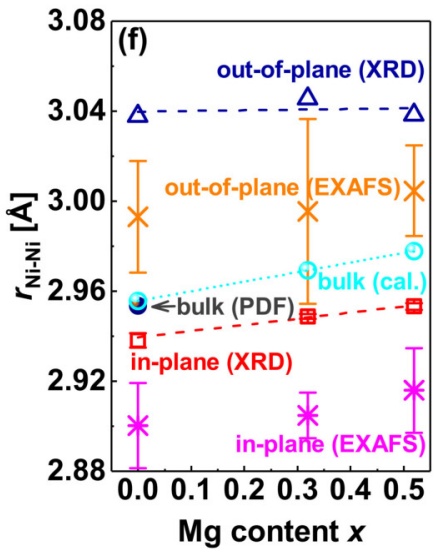

FIG. 1. (a) (111) $d$ spacings $\left(d_{111}^{\text {film }}\right)$ and (b) (11̄1) $d$ spacings $\left(d_{1 \overline{1} 1}^{\text {film }}\right)$ of $\mathrm{Mg}_{x} \mathrm{Ni}_{1-x} \mathrm{O}$ epitaxial thin films as a function of Mg content $(x)$. (c) Lattice deviation $\left(\Delta d_{111}^{\text {film }}\right)$ from ideal cubic structure. (d) Lattice structure of $\mathrm{NiO}$ thin films and atomic arrangements in (111) plane and (101) plane, showing the in-plane nearest-neighbor atomic Ni-Ni distance $\left[r_{\mathrm{Ni}-\mathrm{Ni}}\right.$ (in-plane)], the out-of-plane nearest-neighbor atomic Ni-Ni distance $\left[r_{\mathrm{Ni}-\mathrm{Ni}}\right.$ (out-of-plane)], and the Ni-O nearest-neighbor atomic distance $\left(r_{\mathrm{Ni}-\mathrm{O}}\right)$. (e) $r_{\mathrm{Ni}-\mathrm{O}}$ and (f) in-plane and out-of-plane $r_{\mathrm{Ni}-\mathrm{Ni}}$ obtained from the XRD results of $d_{111}^{\text {film }}$ and $d_{1 \overline{1} 1}^{\text {film }}$ (XRD), as calculated using Vegard's law (cal.) and the powder diffraction file (PDF) of the NiO bulk, and simulated using EXAFS data (EXAFS).

the electron yield measurements of the $\mathrm{O} K$-edge features is typically in the 20-40 ̊ range.

\section{RESULTS AND DISCUSSION}

\section{A. Lattice distortion}

The thickness values of the $\mathrm{Mg}_{x} \mathrm{Ni}_{1-x} \mathrm{O}$ thin films were determined to be $400 \pm 30 \AA$ from the interference fringes recorded in $\mathrm{X}$-ray reflectivity curves and were almost the same as the results that were obtained from the stylus profiler. High-resolution XRSM was used to analyze the epitaxial relationship between each thin film and its substrate. The epitaxial growth of single-crystal $\mathrm{Mg}_{x} \mathrm{Ni}_{1-x} \mathrm{O}$ films on the sapphire substrates was verified using azimuth $\phi$ scans. The crystallographic relationships were determined as follows: $\mathrm{Mg}_{x} \mathrm{Ni}_{1-x} \mathrm{O}$ [12 1 ] $\|$ sapphire [1120] and $\mathrm{Mg}_{x} \mathrm{Ni}_{1-x} \mathrm{O}$ [111] $\|$ sapphire [0001]. The $\phi$ scan of the $\mathrm{Mg}_{x} \mathrm{Ni}_{1-x} \mathrm{O}$ (11) plane shows sixfold symmetry rather than the conventional threefold symmetry of the bulk. This has occurred because of the coexistence of two types of mirror-symmetrical atomic planes in the sapphire substrate, in accordance with the $2.2 \AA$ step height in the sapphire [0001] direction [23].

The 111 and $1 \overline{1} 1$ reciprocal lattice spots that were obtained were then used to estimate the corresponding $d$ spacings. Figures 1(a) and 1(b) show the (111) and (1111) $d$ spacings of the $\mathrm{Mg}_{x} \mathrm{Ni}_{1-x} \mathrm{O}$ thin films $\left(d_{111}^{\text {film }}\right.$ and $\left.d_{1 \overline{1} 1}^{\text {film }}\right)$ as a function of the $\mathrm{Mg}$ content $x$, respectively. Regardless of the $\mathrm{Mg}$ content of the film, however, $d_{111}^{\text {film }}$ is greater than $d_{1 \overline{1} 1}^{\text {film }}$, which indicates that these crystal structures deviate from the ideal cubic structure. For a $\mathrm{NiO}$ thin film, the experimental $d_{111}^{\text {film }}$ value of $\mathrm{NiO}$ (approximately $2.480 \AA$ ) is greater than the corresponding value of the bulk cubic structure $d_{111}^{\text {bulk }}$ [approximately $2.412 \AA$, according to powder diffraction file (PDF) database card no.

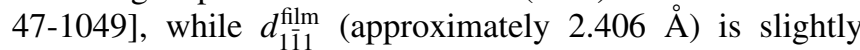
smaller than the corresponding value of the bulk cubic $\mathrm{NiO}$ crystal. As the Mg dopant content increased, the out-of-plane

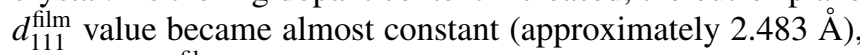

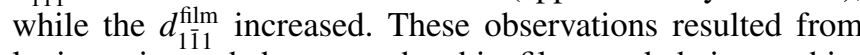
lattice mismatch between the thin films and their sapphire

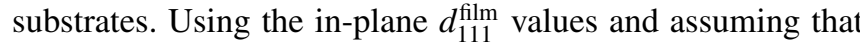
the films have roughly cubic structures, we then estimated the lattice mismatch values between the $\mathrm{Mg}_{x} \mathrm{Ni}_{1-x} \mathrm{O}$ films and the underlying sapphire substrates using domain matching epitaxy theory and based on an integral multiple of the lattice planes $\left[\left(3 d_{1 \overline{2} 1}^{\text {film }}-2 d_{11 \overline{2} 0}^{\text {substrate }}\right) /\left(2 d_{11 \overline{2} 0}^{\text {substrate }}\right)\right]$ [23], which gave values of $7.3 \%, 7.7 \%$, and $7.9 \%$ for $\mathrm{Mg}_{x} \mathrm{Ni}_{1-x} \mathrm{O}$ with $x=0,0.32$, and 0.52 , respectively. All these values were positive, indicating that in-plane compressed stress is present at the interface, which leads to the higher $d_{111}^{\text {film }}$ and lower $d_{1 \overline{1} 1}^{\text {film }}$. Using the $d_{111}^{\text {film }}$ and $d_{1 \overline{1} 1}^{\text {film }}$ values, we can also estimate the deviation of these structures from the ideal cubic structure $\left(\Delta d_{111}^{\text {film }}\right)$, as follows:

$$
\Delta d_{111}^{\mathrm{film}}=-\frac{d_{1 \overline{1} 1}^{\mathrm{film}}-d_{111}^{\mathrm{film}}}{d_{111}^{\mathrm{film}}} .
$$


Figure 1(c) shows that the deviation $\Delta d_{111}^{\text {film }}$ decreases with increasing $\mathrm{Mg}$ content, which means that an increase in the $\mathrm{Mg}$ content can reduce the distortion of the pseudocubic structure.

To enable analysis of the electronic structure in Sec. III D, we must first determine the $\mathrm{NiO}_{6}$ octahedron structure. As discussed above, the lattice of these thin films expands along the [111] direction while shrinking along the [111] direction. The typical octahedron of $\mathrm{Ni}$ with six coordinated $\mathrm{O}$ atoms in the rock-salt structure distorts, and the six $\mathrm{Ni}-\mathrm{O}$ distances remain the same as each other. However, for the central $\mathrm{Ni}$ atom, the 12 nearest $\mathrm{Ni}$ atoms are separated into two groups: six atoms are located at in-plane positions while the other six are in out-of-plane positions. We calculated the nearest atomic distances of $\mathrm{Ni}-\mathrm{O}$ and $\mathrm{Ni}-\mathrm{Ni}\left(r_{\mathrm{Ni}-\mathrm{O}}\right.$ and $\left.r_{\mathrm{Ni}-\mathrm{Ni}}\right)$ using the values of $d_{111}^{\text {film }}$ and $d_{1 \overline{1} 1}^{\text {film }}$, as indicated in Fig. 1(d). The calculated values are shown in Figs. 1(e) and 1(f). The three out-of-plane $r_{\mathrm{Ni}-\mathrm{O}}$ values should be influenced by the stress along the out-of-plane stretching and in-plane compression directions, which is similar to $d_{1 \overline{1} 1}^{\text {film }}$. For comparison with the corresponding bulk materials, we then calculated $r_{\mathrm{Ni}-\mathrm{O}}$ and $r_{\mathrm{Ni}-\mathrm{Ni}}$ on the basis of Vegard's law using radii of 0.72 and $0.69 \AA$ for $\mathrm{Mg}^{2+}$ and $\mathrm{Ni}^{2+}$ [10], respectively, while assuming that $\mathrm{Mg}$ are substituted into the $\mathrm{Ni}$ sites, which will be confirmed in detail in Sec. III C. For the pure $\mathrm{NiO}$ bulk, we obtained values of 2.090 and $2.956 \AA$, which are comparable with the values of 2.089 and $2.954 \AA$ from the PDF card, respectively. This proves that Vegard's law is reliable when used to determine the lattice parameters of the bulk materials here. The calculated $r_{\mathrm{Ni}-\mathrm{O}}$ for the bulk increases from 2.090 to $2.106 \AA$ and $r_{\mathrm{Ni}-\mathrm{Ni}}$ for the bulk increases from 2.956 to $2.978 \AA$, as shown in Figs. 1(e) and 1(f). The calculated atomic distance expands by $0.7 \%$ and is thus roughly consistent with the lattice expansion of $0.7 \%$ in the in-plane direction, which tends to confirm the substitution of $\mathrm{Mg}$ in the $\mathrm{Ni}$ sites.

\section{B. Debye temperature}

The above discussion shows that the doped Mg plays an important role in the lattice distortion of the $\mathrm{Mg}_{x} \mathrm{Ni}_{1-x} \mathrm{O}$ films. To describe the resulting atomic disorder quantitatively, we attempted to evaluate the Debye temperature $\left(\Theta_{M}\right)$ and the Debye-Waller factor $\left(D_{i}^{w}\right)$ of these $\mathrm{Mg}_{x} \mathrm{Ni}_{1-x} \mathrm{O}$ films from the X-ray diffraction intensities of the $1 \overline{1} 1 \mathrm{Bragg}$ points [28]. The relationship between $\Theta_{M}$ and $D_{i}^{w}$ has been described as [29]

$$
\begin{gathered}
D_{i}^{w}=\exp \left(-2 B_{i} \frac{\sin ^{2} \theta}{\lambda^{2}}\right), \\
B_{i}=8 \pi^{2} \overline{u^{2}}, \\
\overline{u^{2}}=\frac{3 \hbar^{2} T}{m k_{B} \Theta_{M}}\left[\frac{1}{x} \int_{0}^{x} \frac{y}{e^{y}-1} d y+\frac{x}{4}\right], \quad x=\frac{\Theta_{M}}{T} .
\end{gathered}
$$

Here the $B$ factor $B_{i}$ is related to $\Theta_{M}, \theta$ is the diffraction angle, $\overline{u^{2}}$ is the mean-square displacement of the lattice point, $m$ is the atomic mass, $k_{B}$ is the Boltzmann constant, $\hbar$ is the Planck constant, and $T$ is the absolute temperature. We defined the real Debye-Waller factor $D_{1}^{w}(=0.81)$ after we obtained $B_{1}$ using Eq. (7) from Ref. [28] and the average integrated intensity of the $1 \overline{1} 1$ and $2 \overline{2} 2$ Bragg peaks [28], which is suitable for the
TABLE I. Parameters of $\mathrm{Mg}_{x} \mathrm{Ni}_{1-x} \mathrm{O}$ thin films at $298 \mathrm{~K}$.

\begin{tabular}{lcccr}
\hline \hline $\mathrm{Mg}_{x} \mathrm{Ni}_{1-x} \mathrm{O}$ & $B_{1}$ or $B_{2}\left(\AA^{2}\right)$ & $\sqrt{\bar{u}^{\mathrm{a}}}(\AA)$ & $d_{1 \overline{1} 1}^{\mathrm{film}}(\AA)$ & $r^{\mathrm{b}}(\%)$ \\
\hline$x=0$ & $2.4(10)$ & $0.18(4)$ & $2.399(3)$ & $7.3(18)$ \\
$x=0.32$ & $3.3(18)$ & $0.20(5)$ & $2.408(1)$ & $8.5(22)$ \\
$x=0.52$ & $8.3(15)$ & $0.32(3)$ & $2.41(1)$ & $13.4(12)$ \\
\hline \hline
\end{tabular}

${ }^{\mathrm{a}}$ Root mean square amplitude of atomic vibration.

${ }^{\mathrm{b}}$ Ratio of $\sqrt{\overline{u^{2}}}$ to $d_{1 \overline{1} 1}^{\text {film }}$.

$\mathrm{NiO}$ films without consideration of the atomic-scale disorder. To estimate the influence of the atomic-scale disorder that is caused by $\mathrm{Mg}$ doping on the $\mathrm{x}$-ray diffraction intensities of the $\mathrm{Mg}_{x} \mathrm{Ni}_{1-x} \mathrm{O}$ thin films, we proposed a pseudo-Debye-Waller factor $D_{2}^{w}$ based on the x-ray $1 \overline{1} 1$ intensity ratio between $\mathrm{Mg}$-doped and undoped $\mathrm{NiO}$ thin films. In this parameter, the structural disorder can be neglected if we assume that all the $\mathrm{Mg}^{2+}$ substitute for $\mathrm{Ni}^{2+}$. We then evaluated our proposed factor $B_{2}$, which in this case includes two values for the $\mathrm{Mg}_{0.32} \mathrm{Ni}_{0.68} \mathrm{O}$ and $\mathrm{Mg}_{0.52} \mathrm{Ni}_{0.48} \mathrm{O}$ epitaxial thin films, using Eq. (10) from Ref. [28]. The results are listed in Table I. The corresponding values of $\Theta_{M}$ from this evaluation are plotted in Fig. 2. The deviations are greater for lower $\mathrm{Mg}$ contents, which is explained by the mathematical solution for $\Theta_{M}$ when using $D_{i}^{w}$ in Eq. (2). In the logarithmic function, there must be a higher $\Theta_{M}$ deviation for a lower $D_{i}^{w}$ value. The root mean square (rms) amplitude $\sqrt{\bar{u}^{2}}$ of the atomic vibration increased with increasing $\mathrm{Mg}$ content, as shown in Table I. The ratio $r$ between the rms amplitude of the atomic vibration and the (1111) $d$ spacing of the $\mathrm{Mg}_{x} \mathrm{Ni}_{1-x} \mathrm{O}$ thin films $d_{1 \overline{1} 1}$ also increased. These values are greater than the corresponding value $(4.6 \%)$ of the ideal bulk $\mathrm{NiO}$ [30], which means that the stress at the film/substrate interface causes greater static atomic displacement than occurs in the bulk and the Mg doping simply enhances this behavior.

To consider the atomic disorder between the $\mathrm{NiO}$ film and the $\mathrm{Mg}_{x} \mathrm{Ni}_{1-x} \mathrm{O}$ film, let us assume that the relationship between $D_{2}^{w}$ and $D_{1}^{w}$ can be described using the atomic order

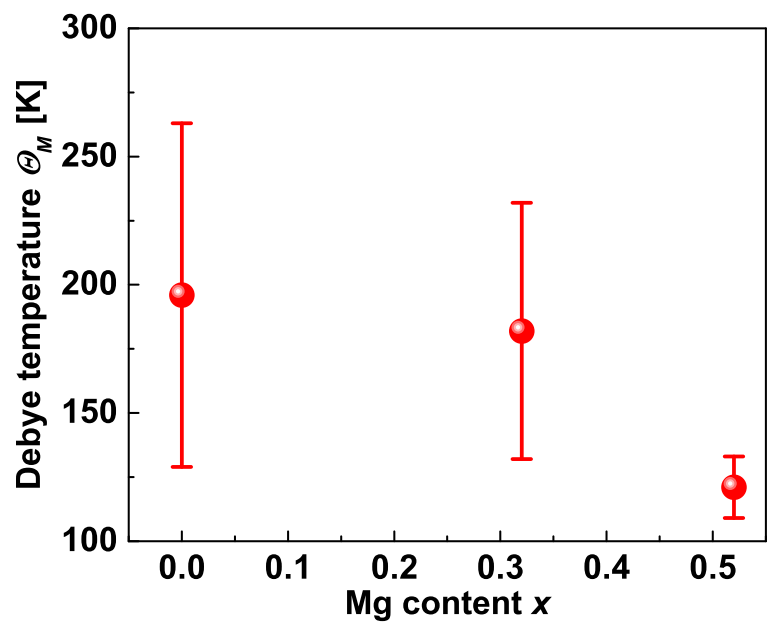

FIG. 2. Debye temperature $\Theta_{M}$, determined from the intensities of the $1 \overline{1} 1 \mathrm{Bragg}$ points, as a function of $\mathrm{Mg}$ content $x$ in $\mathrm{Mg}_{x} \mathrm{Ni}_{1-x} \mathrm{O}$ epitaxial thin films. 
TABLE II. Debye-Waller factor $D_{1}^{w}$ or $D_{2}^{w}$ and atomic order parameters $\exp \delta$ of $\mathrm{Mg}_{0.32} \mathrm{Ni}_{0.68} \mathrm{O}$ and $\mathrm{Mg}_{0.52} \mathrm{Ni}_{0.48} \mathrm{O}$ thin films compared with those of $\mathrm{NiO}$ thin films.

\begin{tabular}{llll}
\hline \hline $\mathrm{Mg}_{x} \mathrm{Ni}_{1-x} \mathrm{O}$ & $D_{1}^{w}$ or $D_{2}^{w}$ & $\exp \delta_{A}$ & \multicolumn{1}{c}{$\exp \delta_{B}$} \\
\hline$x=0$ & $0.81(8)$ & & \\
$x=0.32$ & $0.75(11)$ & $0.93(7)$ & $0.81(12)$ \\
$x=0.52$ & $0.49(6)$ & $0.61(3)$ & $0.53(7)$ \\
\hline \hline
\end{tabular}

parameter $\exp \delta_{A}$, which can be expressed as follows:

$$
\exp \delta_{A}=D_{2}^{w} / D_{1}^{w}
$$

$\exp \delta_{A}$ here stands for the structural order compared with a $\mathrm{NiO}$ film prepared using a growth method similar to that described above. For reference, we compare $D_{i}^{w}$ with the bulk equivalent $D_{\text {bulk }}^{w}(=0.92)$, which was estimated based on $B_{\text {bulk }}$ using Eqs. (2) and (3) for $\Theta_{M}=317.4 \mathrm{~K}$ in Ref. [30]. We also define another order parameter $\exp \delta_{B}$ as follows:

$$
\exp \delta_{B}=D_{2}^{w} / D_{\text {bulk }}^{w} .
$$

Table II lists the values of $D_{i}^{w}$ that were obtained using the $B_{i}$ factors, $\exp \delta_{A}$, and $\exp \delta_{B}$. We found that $\exp \delta_{A}$ had values of 0.93 and 0.61 for $\mathrm{Mg}_{x} \mathrm{Ni}_{1-x} \mathrm{O}$ with $x=0.32$ and 0.52 , respectively. In general, the Debye-Waller factor includes the influence of the thermal disorder along with that of the static atomic disorder. The calculated atomic order parameters $\exp \delta_{A}$ and $\exp \delta_{B}$ both decrease for the $\mathrm{Mg}_{x} \mathrm{Ni}_{1-x} \mathrm{O}$ films as their $\mathrm{Mg}$ content increases. The disorder may include the combined effects of the thermal atomic vibration and the static atomic displacement that were caused by $\mathrm{Mg}$ doping, which are both enhanced in heavily $\mathrm{Mg}$-doped $\mathrm{NiO}$ thin films.

The lattice mismatch and the atomic disorder always affect the crystal domain size and the crystal mosaicity. The domain size of the $\mathrm{Mg}_{x} \mathrm{Ni}_{1-x} \mathrm{O}$ thin films decreased with increasing $\mathrm{Mg}$ content. In the in-plane (out-of-plane) direction, the domain sizes are 167 (105), 89 (69), and 80 (59) $\AA$ for films with $x=0,0.32$, and 0.52 , respectively. The in-plane crystal domain sizes are larger than the corresponding out-of-plane sizes because of the Stranski-Krastanov growth [31] of $\mathrm{NiO}$ (111) planes on the sapphire substrates. In addition, the crystallographic mosaic spread angles (which are $1.12^{\circ}, 1.95^{\circ}$, and $2.74^{\circ}$ for films with $x=0,0.32$, and 0.52 , respectively) also increased with increasing $\mathrm{Mg}$ content. All the changes in the domain size and the mosaic spread angle correspond to the increased lattice mismatch and atomic disorder that is induced by the $\mathrm{Mg}$ doping, which destroys the homogenous microstructure of the epitaxial thin films.

When compared with the $\mathrm{Li}_{x} \mathrm{Ni}_{1-x} \mathrm{O}$ epitaxial films that were studied in our previous work [32], the lattice distortion of the $\mathrm{Mg}_{x} \mathrm{Ni}_{1-x} \mathrm{O}$ films is much smaller. Because of the phase transformation from the rhombohedral structure with random $\mathrm{Li}^{+}$occupation to the hexagonal structure with the preferred occupation of the two cationic sites in $\mathrm{Li}_{x} \mathrm{Ni}_{1-x} \mathrm{O}$ when $x>0.31$ [11,12], the lattice parameter of $\mathrm{Li}_{0.48} \mathrm{Ni}_{0.52} \mathrm{O}$ bulk decreases by $2.3 \%$ [12]. This was confirmed by the reduction of $r_{\text {Ni-O }}$ by $2.5 \%$, based on the measured $d_{111}^{\text {film }}$ of the $\mathrm{Li}_{0.48} \mathrm{Ni}_{0.52} \mathrm{O}$ thin film [32]. In contrast, $\mathrm{Mg}_{x} \mathrm{Ni}_{1-x} \mathrm{O}$ offers higher $\mathrm{Mg}$ solubility with lower lattice distortion. It is difficult to visualize the transformation of the lattice structures of these films with increasing $\mathrm{Mg}$ content. The calculated atomic order parameter of $\mathrm{Mg}_{0.52} \mathrm{Ni}_{0.48} \mathrm{O}(0.61)$ is smaller than that of $\mathrm{Li}_{0.5} \mathrm{Ni}_{0.5} \mathrm{O}$ (0.66) [28], which demonstrates the increased effect of the $\mathrm{Mg}^{2+}$ ions when compared with that of the $\mathrm{Li}^{+}$on the atomic-scale disorder. This may imply that the preferred occupation of some of the cationic sites when the Mg content is high enough is sufficient to form an ordered solid solution similar to that of $\mathrm{Li}_{x} \mathrm{Ni}_{1-x} \mathrm{O}$ with $x>0.31$. In contrast to the nonmonotonic tendency that was observed for the phase transformation in $\mathrm{Li}_{x} \mathrm{Ni}_{1-x} \mathrm{O}$ epitaxial films, the lattice distortion that was induced by the $\mathrm{Mg}^{2+}$ ions resulted in monotonic changes in the microstructural parameters of the $\mathrm{Mg}_{x} \mathrm{Ni}_{1-x} \mathrm{O}$ films, such as the domain size and the mosaic spread.

\section{Local atomic structure}

Figure 3(a) shows the $k^{3}$ weighted EXAFS spectra $k^{3} \chi(k)$ of the Ni $K$ edge of the $\mathrm{Mg}_{x} \mathrm{Ni}_{1-x} \mathrm{O}$ thin films and the $\mathrm{NiO}$ bulk. The EXAFS spectra obtained show clear oscillation in a wide $k$-space range of up to $14 \AA^{-1}$ for all samples. Noticeable differences among the EXAFS spectra of the three thin films due to the increase in $\mathrm{Mg}$ content are clearly visible. Figures 3(b)-3(e) show the Fourier transforms of $k^{3} \chi(k)$ in the $R$-space range of 1.5-3.5 $\AA$ for further analysis. The first peak is due to the interactions with the oxygen atoms $(R \approx 2.1 \AA)$ and the second is caused by the nickel atoms $(R \approx 3.0 \AA)$. The oscillation patterns of the two $\mathrm{Mg}_{x} \mathrm{Ni}_{1-x} \mathrm{O}$ thin films with $x=0.32$ and $x=0.52$ are very similar to that of the pure $\mathrm{NiO}$ thin film (where $x=0$ ) in the spectra. Therefore, the EXAFS results, in conjunction with the XRD results, imply that the $\mathrm{Mg}$ atoms are substituted for the Ni atoms.

To evaluate the local atomic structure, we need to analyze the EXAFS spectra in combination with the simulation results. All contributions to the EXAFS were analyzed using the single-scattering approximation [33]. A scattering amplitude was extracted from simulations of the experimental $\mathrm{Ni}$ foil data and the amplitude value is 0.8. Phase shift functions for both the Ni-O and Ni-Ni distances were calculated in the $\mathrm{NiO}$ rock-salt structure with symmetry of $F m \overline{3} m$ and lattice parameter $a_{0}=4.177 \AA$ (from PDF card 47-1049). There are two occupation possibilities for the doped $\mathrm{Mg}$ atoms, i.e., in the interstitial sites or in the Ni sites, when we assume that the $\mathrm{Mg}$ atoms are distributed randomly in the $\mathrm{NiO}$ rock-salt structure. In the interstitial model, the $\mathrm{Mg}$ atoms occupy the $(1 / 4,1 / 4,1 / 4)$ sites according to the $\mathrm{Mg} / \mathrm{Ni}$ atomic ratio. The spectra were simulated from the first single scattering of the three coordination shells, including the $\mathrm{O}$ atoms, the $\mathrm{Ni}$ atoms, and the interstitial $\mathrm{Mg}$ atoms. The simulated results show that the $\mathrm{Mg}$ atoms deviated from the interstitial sites by $10 \%-20 \%$ for the two $\mathrm{Mg}_{x} \mathrm{Ni}_{1-x} \mathrm{O}(x=0.32,0.52)$ thin films, which differs considerably from the assumed interstitial model. The unsuitability of this model was also evident for larger values of the $R$ factor (which represents the relative error between the fitted result and the data and should be $<2 \%$ [34]), which were too large. In the substitutional model, the $\mathrm{Mg}$ atoms are substituted into Ni sites with the appropriate $\mathrm{Mg} / \mathrm{Ni}$ atomic ratio. We did not distinguish between the $\mathrm{Mg}$ and $\mathrm{Ni}$ coordination shells, in which the $\mathrm{Mg}$ and $\mathrm{Ni}$ atoms are both located at Ni sites. The following EXAFS discussion 

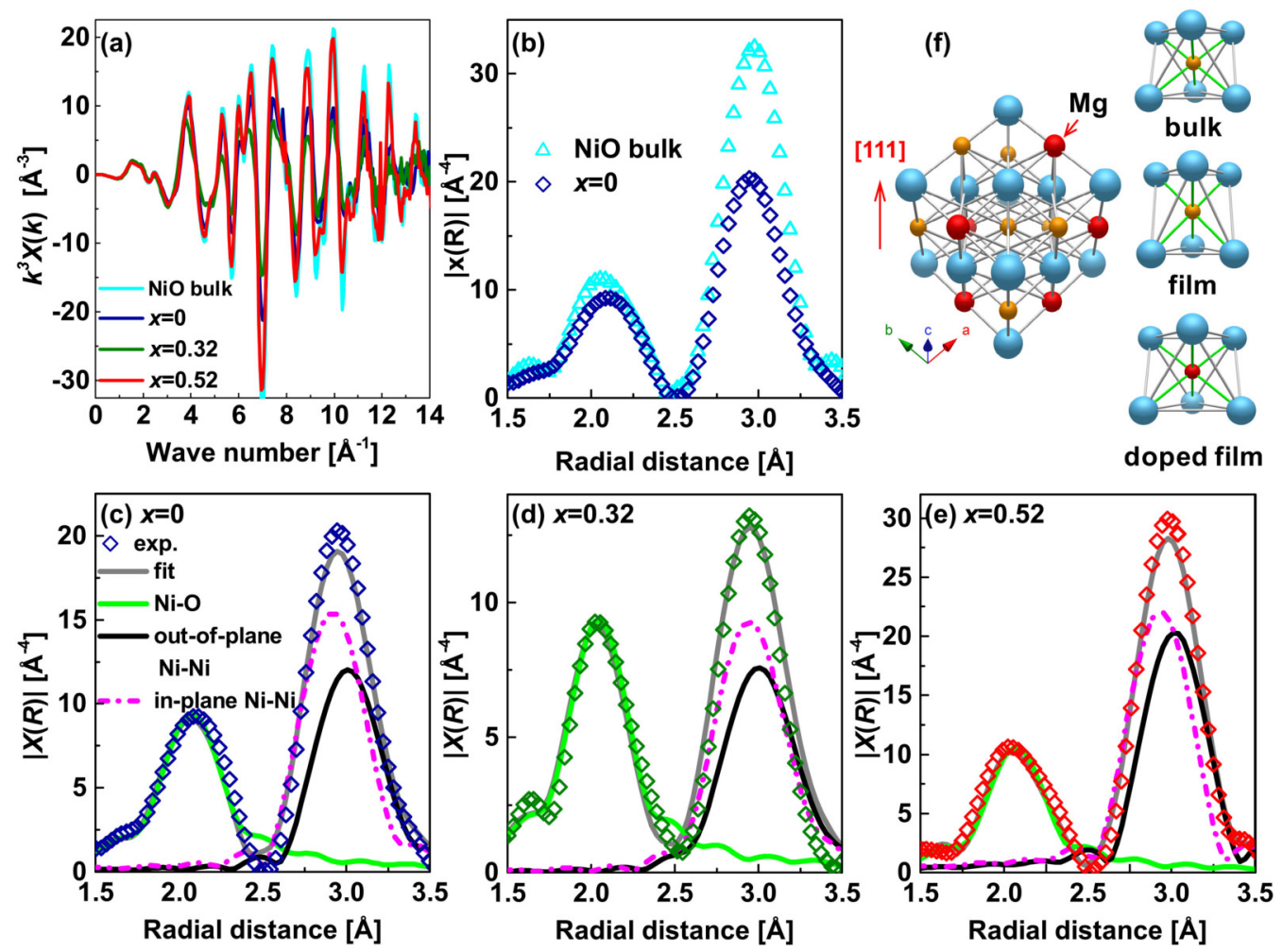

FIG. 3. (a) $k^{3}$ weighted EXAFS spectra $k^{3} \chi(k)$ of the Ni $K$-edge EXAFS in the $\operatorname{Mg}_{x} \mathrm{Ni}_{1-x} \mathrm{O}$ epitaxial thin films and the NiO bulk. (b) Fourier transforms of the $\mathrm{NiO}$ epitaxial thin film and $\mathrm{NiO}$ bulk data. Fourier transforms and their simulation results when using a substitutional model for (c) $x=0$, (d) $x=0.32$, and (e) $x=0.52$. (f) Schematic of $\mathrm{Mg}$ occupation of the (111)-oriented NiO thin film and distortion of the $\mathrm{NiO}_{6}$ octahedra, which was derived from the results shown in Figs. 1 and 3(c)-3(e).

related to $\mathrm{Ni}$ refers to these $\mathrm{Ni}$ sites, and includes both $\mathrm{Ni}$ atoms and substitutional $\mathrm{Mg}$ atoms. Spectra were simulated based on the first single scattering of the $\mathrm{O}$ coordination shells and the $\mathrm{Ni}$-site coordination shells. The fitting between the model and the spectra is good and the simulated results also show the main features of the experimentally observed features. The Ni-O distances $(2.076,2.054$, and $2.074 \AA$, for $x=0,0.32$, and 0.52 , respectively) and $\mathrm{Ni}$ (site)-Ni (site) distances (2.936, 2.942 , and $2.959 \AA$, for $x=0,0.32$, and 0.52 , respectively) that were obtained are almost within the range of values that was calculated based on the XRD results. We can therefore confirm that the $\mathrm{Mg}$ dopant is highly likely to occupy the $\mathrm{Ni}$ sites.

The two models above were both discussed without consideration of the features of the epitaxial thin films. In fact, as shown in Fig. 3(b), there are considerable differences between the $\mathrm{NiO}$ epitaxial thin film and the $\mathrm{NiO}$ bulk in terms of both peak position and shape, and particularly in the peak that corresponds to $r_{\mathrm{Ni}-\mathrm{Ni}}$. The lattice is distorted, as shown by the XRD results in Sec. III A, and thus for the scattering central Ni atom, the six $r_{\mathrm{Ni}-\mathrm{O}}$ out of the (111) plane change synchronously and the six in-plane $r_{\mathrm{Ni}-\mathrm{Ni}}$ and six out-ofplane $r_{\mathrm{Ni}-\mathrm{Ni}}$ change asynchronously. We therefore separate the Ni-site coordination shell into one half in-plane shell and one half out-of-plane shell. The fitted results are presented in Figs. 3(c)-3(e). The simulated nearest atomic distances are plotted in Figs. 1(e) and 1(f). These results are in good agreement with the overall lattice expansion upon an increase in $\mathrm{Mg}$ content in the thin films that were measured using XRD. It is noted that the simulated Ni-O distances do indeed overlap with the values that were obtained from $d_{1 \overline{1} 1}^{\text {film }}$, but not with those obtained from $d_{111}^{\text {film }}$, which includes the out-of-plane stretch only.

In the (111)-oriented $\mathrm{NiO}$ thin film, the atomic layers are composed of stacks of nickel atomic layers and oxygen atomic layers, as illustrated in Fig. 3(f). We have confirmed that $\mathrm{Mg}$ is substituted into the $\mathrm{Ni}$ sites, which always lie in the (111) plane. Because the $\mathrm{Mg}^{2+}$ ions have a slightly larger ionic radii than the $\mathrm{Ni}^{2+}$ ions and because the out-of-plane $\mathrm{Ni}-\mathrm{Ni}$ distance is stretched when compared with that in an ideal cubic rock-salt structure, the $\mathrm{Mg}$ dopant thus causes more lattice distortion along the in-plane direction than along the out-of-plane direction. With the expansion of the lattice caused by the $\mathrm{Mg}$ dopants, distortion of the $\mathrm{NiO}_{6}$ octahedron occurs at the same time, similar to the schematic of the octahedra shown in Fig. 3(f). When compared with the NiO bulk, the three O-Ni-O axes (marked in green) of the octahedra in the $\mathrm{NiO}$ thin films tilt upwards to the [111] direction. In the $\mathrm{Mg}$ doped $\mathrm{NiO}$ films, the three axes tend to tilt back to the normal such that they are perpendicular to each other while the lattice is expanding. In addition to the $\mathrm{Ni}-\mathrm{O}$ atomic distance, the tilt in the axis of the $\mathrm{NiO}_{6}$ octahedron will also affect the overlapping of the electronic orbitals.

\section{Electronic structure}

Because we confirmed both the lattice distortion and the $\mathrm{Mg}$ occupation of the $\mathrm{Mg}_{x} \mathrm{Ni}_{1-x} \mathrm{O}$ thin films above, we will now 

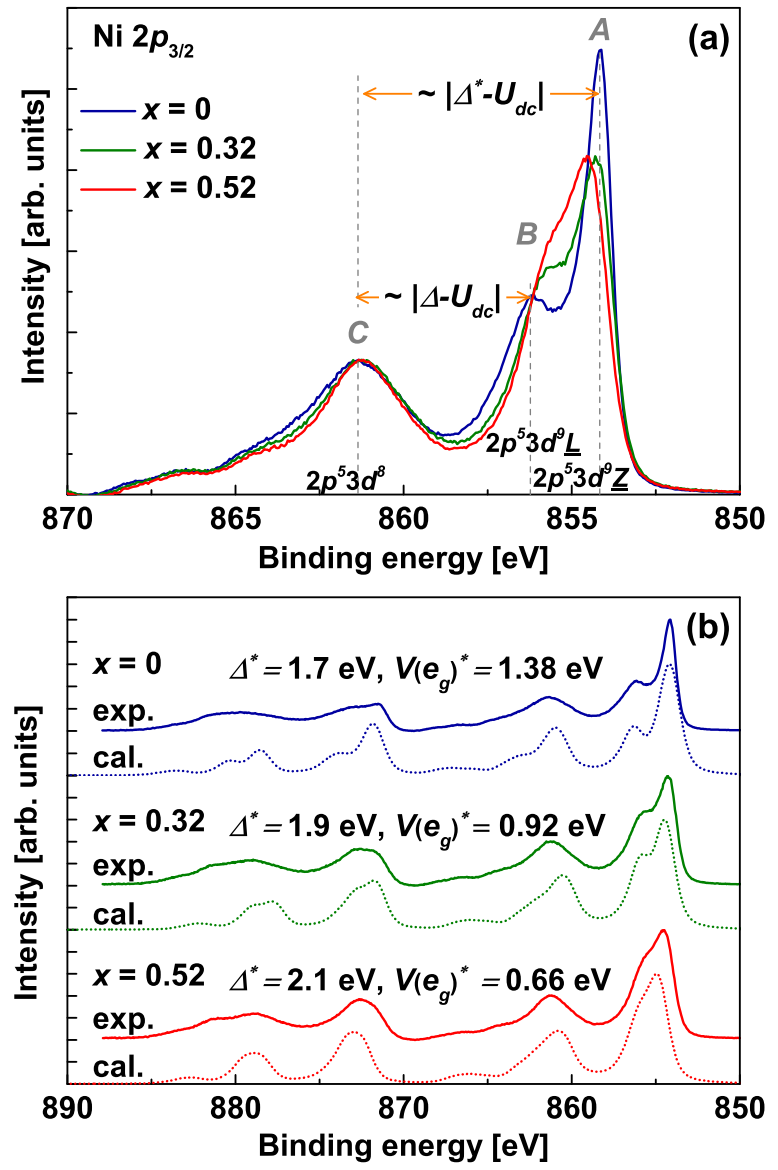

FIG. 4. (a) Core-level spectra of $\mathrm{Ni} 2 p$ for $\mathrm{Mg}_{x} \mathrm{Ni}_{1-x} \mathrm{O}$ with $x=0,0.32$, and 0.52 . Features $A, B$, and $C$ are $2 p^{5} 3 d^{9} \underline{Z}, 2 p^{5} 3 d^{9} \underline{L}$, and $2 p^{5} 3 d^{8}$, respectively, where $\underline{Z}$ and $\underline{L}$ represent a hole in the Zhang-Rice doublet bound states and a hole in $\mathrm{O} 2 p$ ligand states, respectively. $U_{d c}$ is the attractive $\mathrm{O} 2 p$ core-hole potential. $\Delta$ is the conventional charge transfer energy from the $\mathrm{O} 2 p$ states to the $\mathrm{Ni}$ $3 d$ states, and $\Delta^{*}$ is the charge transfer energy from the bound states to the Ni $3 d$ states. (b) Comparison of experimental (exp.) core-level spectra (solid line) with the calculated (cal.) results (dotted line) using an extended configuration interaction model that includes the Zhang-Rice doublet bound states.

focus on the electronic structure of the films. Figure 4(a) shows the Ni $2 p_{3 / 2}$ core-level HAXPES spectra of the $\mathrm{Mg}_{x} \mathrm{Ni}_{1-x} \mathrm{O}$ epitaxial thin films. The characteristic features of $\mathrm{NiO}$ at 854.2, 856.1 , and $861.3 \mathrm{eV}$ are labeled $A, B$, and $C$, respectively. The core-level spectra were normalized with respect to the peak height at the feature $C$. These characteristic features indicate strong electron correlation in the $\mathrm{Ni} 3 d$ states. The intensities and peak positions of features $\mathrm{A}$ and $\mathrm{B}$ are modified by the $\mathrm{Mg}$ doping, which indicates that the electronic structural parameters of the Ni $3 d$ states are modified by the Mg doping. In the previous HAXPES study performed by Taguchi et al. [22], the experimental $\mathrm{Ni} 2 p$ core level spectrum for bulk $\mathrm{NiO}$ was reproduced well via the calculations of the extended configuration interaction (CI) model; this model was based on the single octahedral $\mathrm{NiO}_{6}$ cluster model, in which the additional charge transfer and hybridization that occurred between the Ni $3 d$ and Zhang-Rice doublet bound states was taken into account for treatment of the intersite reaction for simplicity. Because the $\mathrm{Ni} 2 p$ spectrum for the $\mathrm{NiO}$ thin film is almost identical to that of the $\mathrm{NiO}$ bulk, we can therefore discuss the spectral features of the $\mathrm{Mg}_{x} \mathrm{Ni}_{1-x} \mathrm{O}$ thin films by referring to the results of the extended CI calculations. The features $A, B$, and $C$ in the $\mathrm{NiO}$ film predominantly arise from the $2 p^{5} 3 d^{9} \underline{Z}, 2 p^{5} 3 d^{9} \underline{L}$, and $2 p^{5} 3 d^{8}$ photoemission final states, respectively. Here $\underline{Z}$ and $\underline{L}$ represent the Zhang-Rice doublet bound states near the Fermi level and a hole in the $\mathrm{O}$ $2 p$ ligand states, respectively. The energy separation between $A$ and $C$ and the corresponding separation between $B$ and $C$ are given approximately by $\left|\Delta^{*}-U_{d c}\right|$ and $\left|\Delta-U_{d c}\right|$, respectively. Here $U_{d c}, \Delta$, and $\Delta^{*}$ represent the attractive $2 p$ core-hole potential, the charge transfer energy from the $\mathrm{O} 2 p$ and Ni $3 d$ states, and the charge transfer energy from the bound states to the $\mathrm{Ni} 3 d$ states, respectively. The electronic structure values of $\mathrm{U}_{d c}, \Delta$, and $\Delta^{*}$ are 7.7, 4.0, and $1.5 \mathrm{eV}$, respectively. $\Delta^{*}$ and $\Delta$ are both smaller than $U_{d c}$ in $\mathrm{NiO}$, and thus the values of $\Delta^{*}-U_{d c}$ and $\Delta-U_{d c}$ are both negative. From our experimental spectra, we found that $\Delta^{*}$ increased while $\Delta$ decreased with increasing $\mathrm{Mg}$ content. The energy separation between $A$ and $C$ or that between $B$ and $C$ is not only dependent on $\Delta^{*}-U_{d c}$ or $\Delta-U_{d c}$, but is also dependent on the hybridization $V\left(e_{g}\right)^{*}$ between $\mathrm{Ni} 3 d$ and the bound states, or on the $\mathrm{Ni} 3 d-\mathrm{O} 2 p$ hybridization $V\left(e_{g}\right)$. It is therefore difficult to estimate the charge transfer states based on the energy separation of these peaks. Instead, we must use a configuration-interaction model to calculate the relevant parameters based on the HAXPES core-level spectra.

The Ni $2 p$ HAXPES spectra were calculated within the extended configuration-interaction model. This model has been used successfully to analyze the photoemission spectra of various materials; this model has been described in detail previously [22,35-37]. We used the $3 d^{8}, 3 d^{9} \underline{L}, 3 d^{10} \underline{L}^{2}, 3 d^{9} \underline{Z}$, $3 d^{10} \underline{Z}^{2}$, and $3 d^{10} \underline{Z L}$ states in the ground state. We fitted the experimental spectra by simply changing two parameters: $\Delta^{*}$ and $V^{*}$. Apart from these two parameters, all other remaining parameters were fixed and used values taken from previous work: $U_{d d}=6.5 \mathrm{eV}, U_{d c}=7.7 \mathrm{eV}$, the crystal field $10 \mathrm{Dq}=$ $0.3 \mathrm{eV}, \Delta=4.0 \mathrm{eV}$, and $V\left(e_{g}\right)=2.2 \mathrm{eV}$ [22]. The calculated spectra (indicated by the dotted lines) are compared with the experimental spectra (indicated by solid lines) in Fig. 4(b). The typical features are clearly reproduced over the entire energy range. The calculated values of the variable parameters are given as follows: $\Delta^{*}=1.7 \mathrm{eV}$ and $V\left(e_{g}\right)^{*}=1.38 \mathrm{eV}$ for $x=0 ; \Delta^{*}=1.9 \mathrm{eV}$ and $V\left(e_{g}\right)^{*}=0.92 \mathrm{eV}$ for $x=0.32$; and $\Delta^{*}=2.1 \mathrm{eV}$ and $V\left(e_{g}\right)^{*}=0.66 \mathrm{eV}$ for $x=0.52$. When compared with the values of $\Delta^{*}=1.5 \mathrm{eV}$ and $V\left(e_{g}\right)^{*}=$ $1.43 \mathrm{eV}$ for the $\mathrm{NiO}$ single crystal, all the $\mathrm{Mg}_{x} \mathrm{Ni}_{1-x} \mathrm{O}$ epitaxial thin films were found to have larger $\Delta^{*}$ values and smaller $V\left(e_{g}\right)^{*}$ values. This is a result of the expanded lattice structure, which increases the $\mathrm{Ni}-\mathrm{O}$ distance and the $\mathrm{Ni}-\mathrm{Ni}$ distance when compared with the corresponding distance in the single crystal. Due to the weaker intersite interactions, screening from the bound states to $\mathrm{Ni} 3 d$ is suppressed for the epitaxial thin films. Because of the distortion of $\mathrm{NiO}_{6}$ octahedra, the hybridization between $\mathrm{Ni} 3 d$ and the bound states is then weakened.

We have obtained two parameters, $\Delta^{*}$ and $V\left(e_{g}\right)^{*}$, which originate from the Zhang-Rice bound state. The Zhang-Rice bound state is introduced on the basis of the use of $\mathrm{NiO}_{6}$ 


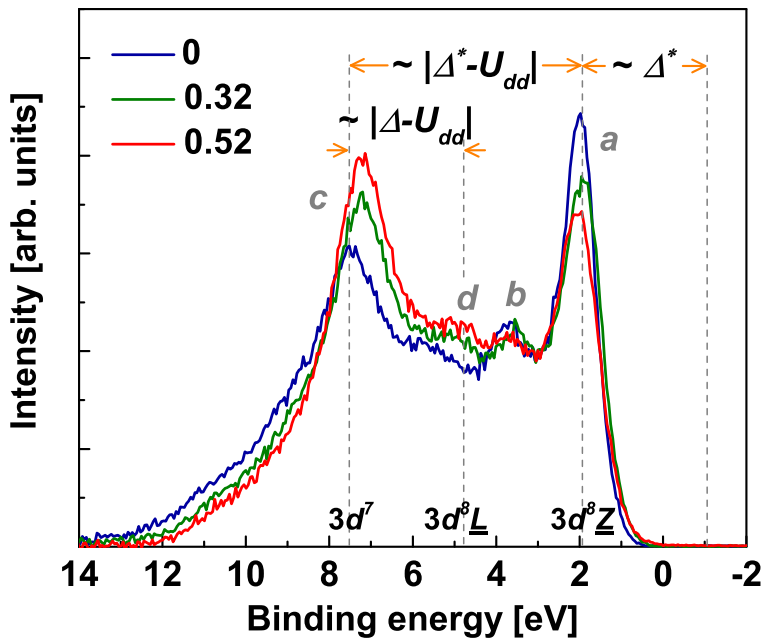

FIG. 5. Valence-band spectra of $\mathrm{Mg}_{x} \mathrm{Ni}_{1-x} \mathrm{O}$ epitaxial thin films with $x=0,0.32$, and $0.52 . U_{d d}$ represents the on-site Coulomb repulsion between the $\mathrm{Ni} 3 d$ states.

octahedra. A hole arises in the ligand orbital of the octahedron and is transferred from the initial ligand to other ligands in the neighboring octahedra [38]. Because the $\mathrm{NiO}_{6}$ octahedra are distorted with stretched atomic distances, the hybridization between $\mathrm{Ni} 3 d$ and $\mathrm{O} 2 p$ is reduced. While the axes of the $\mathrm{NiO}_{6}$ octahedron tilt back towards the ideal position, the hybridization in the octahedron is enhanced, but appears too weak based on the spectra. Additionally, the Mg dopants that were substituted into the $\mathrm{Ni}$ sites disturb the intersite interactions among the octahedra. Here the atomic distance and the dopant substitution strongly affect the final reduction of $2 p^{5} 3 d^{9} \mathrm{Z}$, which is demonstrated by the smaller value of $V\left(e_{g}\right)^{*}$ for more heavily doped thin films. Meanwhile, the reduced $\Delta^{*}$ value for the more heavily doped thin film appears as a larger band gap between the bound state and the unoccupied Ni $3 d$ orbital. [39] The relative shift in the bound state peak is approximately $0.4 \mathrm{eV}$ among the $\mathrm{Mg}_{x} \mathrm{Ni}_{1-x} \mathrm{O}$ thin films with $x$ ranging from 0 to 0.52 .

Figure 5 shows the valence-band HAXPES spectra of the $\mathrm{Mg}_{x} \mathrm{Ni}_{1-x} \mathrm{O}$ epitaxial thin films. Four distinct features, which are denoted by letters $a-d$ are observed in the spectrum for the $\mathrm{NiO}$ thin film. The changes in the valence-band spectra as a function of the $\mathrm{Mg}$ doping content are also consistent with the increased $\Delta^{*}$ and reduced $V\left(e_{g}\right)^{*}$ that were calculated from the core-level spectra shown in Fig. 4(b). Similar to the energy separation between features $A$ and $C$ shown in Fig. 4(a), the separation energy between features $a$ and $c$ that is shown in Fig. 5 is compressed for the more heavily doped films because of the higher value of $\Delta^{*}$. Noticeably, the intensity of feature $a$ is weakened because of a reduction of the Zhang-Rice bound states near the Fermi level in a similar manner to that described above for the Ni $2 p$ core level. Because the density of state (DOS) is mainly dominated by Ni $3 d$ electron near the Fermi level (the main feature $\mathrm{O} 2 p$ is located near $5 \mathrm{eV}$ ) [40], $\Delta^{*}$ can be also used to evaluate the band gap in our spectra approximately. This means that the band gap increases with the increasing $\mathrm{Mg}$ content. Accordingly, the peak intensity near the Fermi level shown in Fig. 5 has reduced. The peak shift

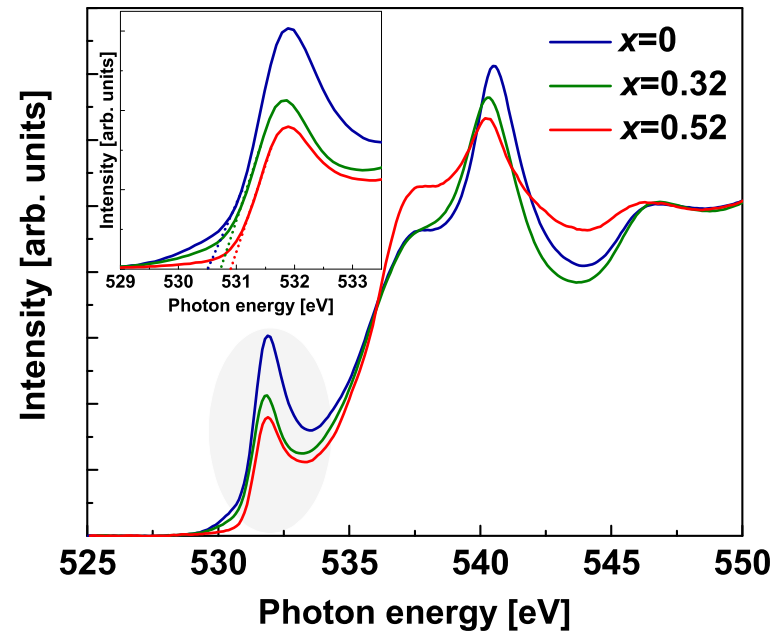

FIG. 6. NEXAFS results for the $\mathrm{O} K$ edge in the $\mathrm{Mg}_{x} \mathrm{Ni}_{1-x} \mathrm{O}$ epitaxial thin films. The inset contains a magnified view of the isolated peaks located near the Fermi level to show the spectral edges.

is, however, less than $0.2 \mathrm{eV}$. The peak therefore may possibly shift more obviously in the unoccupied state.

Thus far we have discussed the occupied states below the Fermi level. For an analysis of the band gap, measurements of the unoccupied states above the Fermi level are also helpful. NEXAFS is a complementary technique that can be used to reveal an unoccupied state in a material and provide the occupation- and symmetry-projected density of states. Figure 6 shows the oxygen $K$-edge NEXAFS spectra of the $\mathrm{Mg}_{x} \mathrm{Ni}_{1-x} \mathrm{O}$ (111) films. The electron excitation occurs from the $\mathrm{O} 1 s$ state to an $\mathrm{O} 2 p$ state. The $\mathrm{O} K$-edge spectra show an isolated peak between 529 and $534 \mathrm{eV}$ and a double-peak structure around 534-544 eV. The features of the $\mathrm{Mg}_{x} \mathrm{Ni}_{1-x} \mathrm{O}$ thin films represent an assignment of the previously observed features of the NiO bulk [41]. The isolated peak has been identified and is attributed to the strong hybridization between the $\mathrm{O} 2 p$ orbitals and the Ni $3 d e_{g}$ orbitals [41,42]. As the $\mathrm{Mg}$ content is increased, the spectral edge near the Fermi level is transferred into the higher binding energy region and its intensity is reduced, thus increasing the band gap by $0.4 \mathrm{eV}$. It is reasonable to suppose that the changes are a result of the direct hybridization, as predicted above based on the values of $\Delta^{*}$. The changes are also a result of the reduced level of charge transfer from the bound state. The features between 534 and $544 \mathrm{eV}$ are mainly attributed to the hybridization between $\mathrm{O} 2 p$ and $\mathrm{Ni} 4 s$. The left shoulder only exists in a transition metal oxide with $3 d$ electrons and thus did not appear for the pure $\mathrm{MgO}[41,43]$. The shoulder became enhanced with increasing Mg content, which signifies the hybrid orbital rearrangement caused by the $\mathrm{Mg}$ dopants. From the increase in the $2 p^{5} 3 d^{8}$ peak in the core level, it is apparent that the occupied and unoccupied electron states are shifted far away from the Fermi level.

\section{E. Band gap}

In Secs. III A and III B we measured the lattice distortion and the atomic disorder in the $\mathrm{Mg}_{x} \mathrm{Ni}_{1-x} \mathrm{O}$ film structure using synchrotron $\mathrm{x}$-ray techniques including XRD and EXAFS. In 


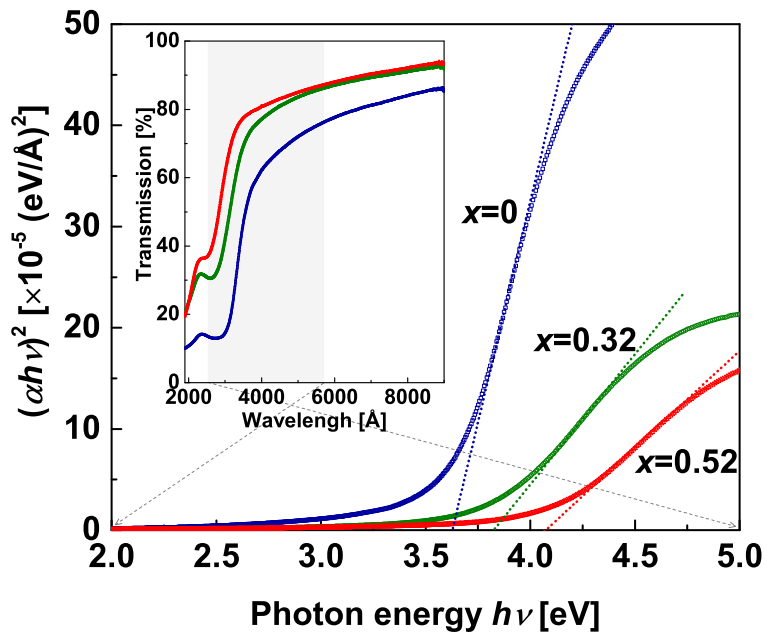

FIG. 7. Absorption edges of $\mathrm{Mg}_{x} \mathrm{Ni}_{1-x} \mathrm{O}$ epitaxial thin films that were estimated from the optical transmittance spectra (see inset), where $\alpha$ is the absorption coefficient. For $x=0,0.32$, and 0.52 , the optical band gaps are $3.63,3.83$, and $4.07 \mathrm{eV}$, respectively.

Sec. III C we confirmed the substitutional Mg occupation of the Ni sites. There are two main factors from the lattice that affect the electronic structure. The first factor is the octahedron distortion, which includes octahedron expansion and other types of deviations from symmetry. Octahedron expansion enhanced the elongation of the nearest $\mathrm{Ni}-\mathrm{O}$ distance, which in turn reduced the hybridization of both $\mathrm{Ni} 3 d$ and $\mathrm{O} 2 p$. The octahedron tilting also influenced the hybridization by tuning the overlap area of $\mathrm{Ni} 3 d$ and the $\mathrm{O} 2 p$ orbital. The other factor is the $\mathrm{Mg}$ dopants that occupied the Ni sites, which influences the Zhang-Rice state intensity and the band gap between the band state and unoccupied $3 d$ state. The electronic features of both the occupied orbitals and the unoccupied orbitals almost appear in the photoelectron spectra and the absorption spectra, respectively, as shown in Sec. III D. The reduced intensities of both the Zhang-Rice state below the Fermi level and the isolated peak above the Fermi level confirmed the reduced electron state that occurred near the Fermi level. At the same time, the edge of the isolated peak was shifted up to a higher energy. The relative shift between the peaks for $\mathrm{Mg}$ contents of $x=0$ and $x=0.52$ is around $0.5 \mathrm{eV}$, which is approximately consistent with the calculated change in $\Delta^{*}$ from 1.7 to $2.1 \mathrm{eV}$.

We estimated the transmittance spectra and obtained both the absorption edge and the band gap of these films, as shown in Fig. 7. Indeed, the transmittance values of the thin films increased and their absorption edges moved towards higher energies with increasing Mg content. Using Tauc's method [44], we plotted the relationship between $(\alpha h v)^{2}$ and the photon energy $h v$. Here $\alpha$ is the absorption coefficient. The intercept of the plot is the band gap, which changed from 3.63 to $4.07 \mathrm{eV}$ as $x$ increased from 0 to 0.52 . These results are also consistent with the previous research on $\mathrm{Mg}_{x} \mathrm{Ni}_{1-x} \mathrm{O}$ films [14-16]. It should be noted that the band gap increases by almost the same amount that was predicted during the calculation of $\Delta^{*}$ and was similar to the values that were measured in NEXAFS. This shows that the band gap is mainly affected by the $\mathrm{Ni} 3 d$ and $\mathrm{O} 2 p$ hybridization in the $\mathrm{NiO}_{6}$ octahedra and the Zhang-Rice bound state.

\section{CONCLUSIONS}

We investigated $\mathrm{Mg}_{x} \mathrm{Ni}_{1-x} \mathrm{O}(0 \leqslant x \leqslant 0.52)$ (111) thin films epitaxially grown on sapphire (0001) substrates. We evaluated the lattice distortion and the atomic order of these films using XRD data. The substitutional occupation of $\mathrm{Ni}$ sites by the Mg dopants was confirmed by EXAFS. We also evaluated the occupied and unoccupied electronic structures using HAXPES and NEXAFS, respectively. With regard to the lattice mismatch between the thin films and the underlying sapphire substrate, the lattice of the $\mathrm{Mg}_{x} \mathrm{Ni}_{1-x} \mathrm{O}$ film was stretched in the out-of-plane [111] direction. This change weakened, however, with increasing $\mathrm{Mg}$ dopant content. The Mg-doped film with $x=0.52$ had a much lower Debye temperature and also a smaller atomic order parameter than the other films. The increasing numbers of $\mathrm{Mg}$ dopants resulted in distortion of the $\mathrm{NiO}_{6}$ octahedra and thus fewer $\mathrm{NiO}_{6}$ octahedra. In combination with the configuration-interaction model calculations, we reproduced the doping dependence of the characteristic features that were observed in the experimental spectra, which indicated that Zhang-Rice doublet bound states on the top of the valence band were strongly dependent on the $\mathrm{Mg}$ doping. The distortion of the $\mathrm{NiO}_{6}$ octahedra directly affects the charge transfer from the band state to the unoccupied state and hybridization of both $\mathrm{Ni} 3 d$ and $\mathrm{O} 2 p$. Mg occupation of the Ni sites leads to a reduction of the nonlocal Zhang-Rice bound state, which was also observed in the dopant dependence of both the core-level and valence band HAXPES spectra. We also explained the novel band gap tuning and attributed it to the Ni $3 d$ and $\mathrm{O} 2 p$ hybridization.

\section{ACKNOWLEDGMENTS}

The thin-film XRD measurements and HAXPES measurements were performed by the NIMS Synchrotron X-ray Station at SPring-8 under Proposals No. 2014B4900, No. 2016B4901, and No. 2016B4906 as part of the NIMS Nanotechnology Platform (Projects No. A-14-NM-0110, No. A-16-NM-0122, and No. A-16-NM-0127) on microstructural characterization, respectively. The XAFS measurements were performed at the SPring-8 under Proposals No. 2016B1029 and No. 2016B1030 and using the SAGA Light Source under Proposal No. 1701129G. This work was also partly supported by Kakenhi Hojyokin [OS: Grants-in-Aid for Basic Research (C), No. 15K04616]. The authors would like to thank Dr. S. Ueda and Dr. Y. Cui for helpful discussions and are also grateful to Dr. K. Yokoyama, Dr. S. Takeda, Professor Y. Kagoshima, and Professor J. Matsui at the University of Hyogo for their technical contributions. The authors would also like to thank Hiroshima Synchrotron Orbital Radiation, Hiroshima University, and JAEA/SPring-8 for the use of the HAXPES setup at the NIMS BL15XU beamline of SPring-8. 
[1] H. Ohta, M. Hirano, K. Nakahara, H. Maruta, T. Tanabe, M. Kamiya, T. Kamiya, and H. Hosono, Appl. Phys. Lett. 83, 1029 (2003).

[2] J. Jung, D. L. Kim, S. H. Oh, and H. J. Kim, Sol. Energ. Mater. Sol. C 102, 103 (2012).

[3] Y. Ren, W. K. Chim, L. Guo, H. Tanoto, J. Pan, and S. Y. Chiam, Sol. Energ. Mater. Sol. C 116, 83 (2013).

[4] R. Deng, B. Yao, Y. F. Li, Y. Xu, J. C. Li, B. H. Li, Z. Z. Zhang, L. G. Zhang, H. F. Zhao, and D. Z. Shen, J. Lumin. 134, 240 (2013).

[5] W. L. Jang, Y. M. Lu, W. S. Hwang, and W. C. Chen, J. Eur. Ceram. Soc. 30, 503 (2010).

[6] S. Nandy, U. N. Maiti, C. K. Ghosh, and K. K. Chattopadhyay, J. Phys.: Condens. Matter 21, 115804 (2009).

[7] J. Wu, C. W. Nan, Y. Lin, and Y. Deng, Phys. Rev. Lett. 89, 217601 (2002).

[8] Y. H. Lin, J. Wang, J. Cai, M. Ying, R. Zhao, M. Li, and C. W. Nan, Phys. Rev. B 73, 193308 (2006).

[9] S. Hufner, Adv. Phys. 43, 183 (1994).

[10] D. R. Lide, CRC Handbook of Chemistry and Physics (CRC, Boca Raton, FL, 1998).

[11] C. B. Azzoni, A. Paleari, V. Massarotti, M. Bini, and D. Capsoni, Phys. Rev. B 53, 703 (1996).

[12] J. B. Goodenough, D. G. Wickham, and W. J. Croft, J. Phys. Chem. Solids 5, 107 (1958).

[13] H. Nishitani, K. Ohta, S. Kitano, R. Hamano, M. Inada, T. Shimizu, S. Shingubara, H. Kozuka, and T. Saitoh, Appl. Phys. Express 8, 105801 (2015).

[14] R. C. Boutwell, M. Wei, A. Scheurer, J. W. Mares, and W. V. Schoenfeld, Thin Solid Films 520, 4302 (2012).

[15] Y. H. Kwon, S. H. Chun, and H. K. Cho, Thin Solid Films 529, 417 (2013)

[16] Y. Guo, L. Zhu, J. Jiang, Y. Li, L. Hu, H. Xu, and Z. Ye, Thin Solid Films 558, 311 (2014).

[17] F. H. Herbstein, Adv. Phys. 10, 313 (1961).

[18] S. L. Quimby and P. M. Sutton, Phys. Rev. 91, 1122 (1953).

[19] C. A. Niedermeier, M. Rasander, S. Rhode, V. Kachkanov, B. Zou, N. Alford, and M. A. Moram, Sci. Rep. 6, 31230 (2016).

[20] G. A. Sawatzky, and J. W. Allen, Phys. Rev. Lett. 53, 2339 (1984).

[21] M. A. van Veenendaal, and G. A. Sawatzky, Phys. Rev. Lett. 70, 2459 (1993).

[22] M. Taguchi, M. Matsunami, Y. Ishida, R. Eguchi, A. Chainani, Y. Takata, M. Yabashi, K. Tamasaku, Y. Nishino, T. Ishikawa, Y. Senba, H. Ohashi, and S. Shin, Phys. Rev. Lett. 100, 206401 (2008).

[23] R. Yamauchi, Y. Hamasaki, T. Shibuya, A. Saito, N. Tsuchimine, K. Koyama, A. Matsuda, and M. Yoshimoto, Sci. Rep. 5, 14385 (2015).

[24] O. Sakata, M. S. Yi, A. Matsuda, J. Liu, S. Sato, S. Akiba, A. Sasaki, and M. Yoshimoto, Appl. Surf. Sci. 221, 450 (2004).
[25] L. S. R. Kumara, O. Sakata, A. Yang, R. Yamauchi, M. Taguchi, A. Matsuda, and M. Yoshimoto, J. Chem. Phys. 141, 044718 (2014).

[26] S. Tanuma, C. J. Powell, and D. R. Penn, Surf. Interface Anal. 43, 689 (2011).

[27] B. Ravel and M. Newville, J. Synchrotron Radiat. 12, 537 (2005).

[28] A. Yang, O. Sakata, R. Yamauchi, L. S. R. Kumara, C. H. Song, Y. Katsuya, A. Matsuda, and M. Yoshimoto, J. Appl. Crystallogr. 48, 1896 (2015).

[29] R. W. James, The Optical Principles of The Diffraction of $X$-rays (G. Bell and Sons, London, 1962).

[30] R. Freer, J. Mater. Sci. 16, 3225 (1981).

[31] W. Weiss, and M. Ritter, Phys. Rev. B 59, 5201 (1999).

[32] A. Yang, O. Sakata, R. Yamauchi, Y. Katsuya, L. S. R. Kumara, Y. Shimada, A. Matsuda, and M. Yoshimoto, Appl. Surf. Sci. 320, 787 (2014).

[33] A. Anspoks, A. Kalinko, R. Kalendarev, and A. Kuzmin, Phys. Rev. B 86 17, 174114 (2012).

[34] W. A. Steen, S. W. Han, Q. Yu, R. A. Gordon, J. O. Cross, E. A. Stern, G. T. Seidler, K. M. Jeerage, and D. T. Schwartz, Langmuir 18, 7714 (2002).

[35] M. Taguchi, A. Chainani, S. Ueda, M. Matsunami, Y. Ishida, R. Eguchi, S. Tsuda, Y. Takata, M. Yabashi, K. Tamasaku, Y. Nishino, T. Ishikawa, H. Daimon, S. Todo, H. Tanaka, M. Oura, Y. Senba, H. Ohashi, and S. Shin, Phys. Rev. Lett. 115, 256405 (2015).

[36] M. Taguchi, A. Chainani, M. Matsunami, R. Eguchi, Y. Takata, M. Yabashi, K. Tamasaku, Y. Nishino, T. Ishikawa, S. Tsuda, S. Watanabe, C. T. Chen, Y. Senba, H. Ohashi, K. Fujiwara, Y. Nakamura, H. Takagi, and S. Shin, Phys. Rev. Lett. 104, 106401 (2010).

[37] M. Taguchi, A. Chainani, K. Horiba, Y. Takata, M. Yabashi, K. Tamasaku, Y. Nishino, D. Miwa, T. Ishikawa, T. Takeuchi, K. Yamamoto, M. Matsunami, S. Shin, T. Yokoya, E. Ikenaga, K. Kobayashi, T. Mochiku, K. Hirata, J. Hori, K. Ishii, F. Nakamura, and T. Suzuki, Phys. Rev. Lett. 95, 177002 (2005).

[38] J. Bala, A. M. Oles, and J. Zaanen, Phys. Rev. Lett. 72, 2600 (1994).

[39] M. Taguchi, A. Chainani, N. Kamakura, K. Horiba, Y. Takata, M. Yabashi, K. Tamasaku, Y. Nishino, D. Miwa, T. Ishikawa, S. Shin, E. Ikenaga, T. Yokoya, K. Kobayashi, T. Mochiku, K. Hirata, and K. Motoya, Phys. Rev. B 71, 155102 (2005).

[40] R. Eder, Phys. Rev. B 91, 245146 (2015).

[41] J. G. Chen, Surf. Sci. Rep. 30, 1 (1997).

[42] P. Kuiper, G. Kruizinga, J. Ghijsen, G. A. Sawatzky, and H. Verweij, Phys. Rev. Lett. 62, 221 (1989).

[43] I. Davoli, A. Marcelli, A. Bianconi, M. Tomellini, and M. Fanfoni, Phys. Rev. B 33, 2979(R) (1986).

[44] J. Tauc, R. Grigorovici, and A. Vancu, Phys. Status Solidi 15, 627 (1966). 